\title{
GREGOR WEBER
}

\section{Traum und Alltag in hellenistischer Zeit*}

I.

„Bezeichnend für den Charakter des hellenistischen Zeitalters ist es, dass zwei geile Triebe der griechischen Litteratur üppiger als vorher ins Kraut schossen: Traum- und Kochbücher. Der Reichthum an Schriften über Traumauslegung, die zum Theil hervorragende Denker zu Verfassern hatten, zeigt uns, wie immer drohender der Mysticismus sein Haupt erhebt; das Gegenstück dazu giebt die lange Reihe schriftstellernder Köche: in ihnen verkörpert sich die sinnlichmaterielle Richtung einer entgeisteten Welt." ${ }^{\text {"1 }}$

Mit diesem Dictum formulierte Franz Susemihl in seiner im Jahre 1891 erschienenen und auch heute noch wertvollen „Geschichte der griechischen Litteratur in der Alexandrinerzeit" eine zweifellos zeitbedingte Einstellung zu antiken Traumberichten und zu ihrer Deutung, wie sie auch danach noch lange Zeit verbreitet war - und vielleicht noch ist: ${ }^{2}$ minderwertige Literatur, Ausdruck eines weit verbreiteten 'Aberglaubens', akzeptiert allenfalls zum Amüsement eines gebildeten Publikums, Zeugnis für einen gewaltigen Abstieg gegenüber der Polisreligion in klassischer Zeit. Muß im folgenden die gastronomische Literatur außer acht bleiben, so soll die Aufmerksamkeit über die angesprochenen Träume und ihre Deutung hinaus auch den Träumenden selbst gelten, jeweils für die hellenistische Zeit. Denn sie können wesentliche Einsichten in Fragen von Religion, Alltagsgeschichte und Men-

* Die vorliegenden Überlegungen konnten gemeinsam mit den Ausführungen, die der in Anm. 5 genannten Studie zugrundeliegen, in den althistorischen bzw. altertumswissenschaftlichen Kolloquien der Universitäten Augsburg, Bielefeld, Bonn, Freiburg, Potsdam und Trier sowie bei der Generalversammlung der Görres-Gesellschaft in Mainz vorgetragen werden. Ich danke den dortigen Auditorien für zahlreiche Anregungen, ebenso der Fondation Hardt (Vandoeuvres/Genève) für die Möglichkeit, das Thema während eines Forschungsaufenthaltes vertiefen zu können. Die Vortragsform wurde weitgehend beibehalten, der Text um Quellenbelege und bibliographische Hinweise ergänzt.

${ }^{1}$ F. Susemihl, Geschichte der griechischen Litteratur in der Alexandrinerzeit, 2 Bde., Leipzig 1891/92, hier Bd. 1, S. 868 (Hervorhebung im Original).

${ }^{2}$ Bemerkenswert ist jedenfalls, daß in den Indizes der sog. 'Standardwerke' zum Hellenismus ein Lemma 'Traum' oder auch 'Vision' meist fehlt - so etwa in M. Rostovtzeff, The Social and Economic History of the Hellenistic World, 3 Bde., Oxford 1941 - oder aber Urteile dezidiert negativ oder pauschal sind, etwa M. P. Nilsson, Geschichte der griechischen Religion (HbAW V 2), München ${ }^{3} 1974$, Bd. 2, S. 231; R. Muth, Einführung in die griechische und römische Religion, Darmstadt 1988, S. 199 ff.; H. Bengtson, Die hellenistische Weltkultur, Stuttgart 1988, S. 47 f. Wesentlich vorurteilsfreier ist dagegen der wenig rezipierte Beitrag von Z. Stewart, La religione, in: R. Bianchi Bandinelli (Hg.), Storia e civiltà dei Greci, Bd. 8: La società ellenistica. Economia, diritto, religione, Mailand 1977, S. 503-616, hier S. 598 ff. („astrologia e magia“); grundlegend bleibt immer noch E. R. Dodds, The Greeks and the Irrational, Berkeley, Los Angeles 1951, S. 102-134. 
talität gesellschaftlicher Gruppen vermitteln, ${ }^{3}$ da aussagekräftiges Material in Form von Inschriften und Papyri, nicht zuletzt auch in Bildzeugnissen und literarischen Texten vorliegt: Dieses Material ist seit langem publiziert, jedoch sehr verstreut bzw. in einen anderen Kontext gestellt. ${ }^{4}$

Mein besonderes Interesse gilt dabei nicht den in beachtlicher Zahl vorliegenden Träumen der hellenistischen Herrscher bzw. ihres Umfeldes sowie den möglichen Umständen ihrer Verbreitung, ${ }^{5}$ sondern gewissermaßen deren Voraussetzungen: Es soll der 'traumgläubige' Horizont von gebildeten und weniger gebildeten Zeitgenossen nachgezeichnet und eine Antwort auf folgende Fragen versucht werden: In welchen Situationen wurde von Träumen berichtet? Was sollte damit erklärt werden? Wer waren die Träumenden? Haben sie selbst oder andere Personen aus diesen Träumen Konsequenzen für ihr Alltagsleben gezogen? Es geht mir demzufolge, um etwaige Mißverständnisse auszuschließen, nicht um den Versuch, die Psyche einzelner Persönlichkeiten mit Hilfe ihrer Träume verstehen zu wollen: ${ }^{6}$ Dies ist nicht möglich aufgrund des Fehlens ausreichender biographischer Informationen, der Andersartigkeit antiker und moderner Traumdeutung sowie vor allem der Tatsache, daß die überlieferten Träume selbst immer schon überformt sind bzw. mit einer bestimmten Absicht erzählt wurden.

${ }^{3}$ Zur Definition von 'Mentalität' in religiösem Kontext vgl. H. W. Pleket, Religious History as the History of Mentality, in: H. S. Versnel (Hg.), Faith, Hope, and Worship. Aspects of Religious Mentality in the Ancient World (Studies in Greek and Roman Religion 2), Leiden 1981, S. 152-192, hier 152 f. Neuere Überlegungen zum Begriff 'Mentalität' haben deutlich gemacht, daß nicht von einem einheitlichen Verständnis auszugehen ist: vgl. G. Tellenbach, 'Mentalität', in: FS C. Bauer, Sigmaringen 1974, S. 11-30; O. G. Oexle, Die 'Wirklichkeit' und das 'Wissen'. Ein Blick auf das sozialgeschichtliche Oeuvre von Georges Duby, HZ (= Historische Zeitschrift) 232 (1981), S. 61-91; V. Sellin, Mentalität und Mentalitätsgeschichte, HZ 241 (1985), S. 555-598; F. Graus, Mentalität. Versuch einer Begriffsbestimmung und Methoden der Untersuchung, in: ders. (Hg.), Mentalitäten im Mittelalter. Methodische und inhaltliche Probleme (Vorträge und Forschungen 35), Sigmaringen 1987, S. 9-48. Im folgenden ist Mentalität verstanden im Sinne einer für eine größere oder kleinere Gruppe typischen Verhaltensweise, die nicht weiter hinterfragt zu sein scheint.

${ }^{4}$ Die papyrologischen Zeugnisse wurden bereits zusammengestellt von D. del Corno, Contributi papirologici allo studio dell'onirocritica, Atti del IX congresso internazionale di papirologia, Mailand 1966, S. 109-117; jüngst auch V. Leuci, Dream-technical Terms in the Greco-Roman World, Diss. University of Missouri/Columbia 1993, S. 47 ff.; einige allgemeine Hinweise bei A. H. M. Kessels, Dreams in Apollonius'Argonautica, in: J. den Boeft/ders. (Hg.), Arctus. Studies in Honour of H. L. W. Nelson, Utrecht 1982, S. 155 f. Dagegen behandelt der Abschnitt über Träume von L. H. Martin, Hellenistic Religions. An Introduction, New York, Oxford 1987, S. 48-52, entgegen der Formulierung des Titels nur Artemidor, Aelius Aristides sowie Beispiele für die Inkubation aus der Zeit vor dem Hellenismus.

${ }^{5}$ Diese sind Gegenstand einer eigenen Studie, die im Jahre 1999 in Heft 1 der Zeitschrift „Archiv für Kulturgeschichte“ unter dem Titel „Herrscher und Traum in hellenistischer Zeit" erscheinen wird. Das Material aus der römischen Kaiserzeit behandle ich in meiner Habilitationsschrift, die in diesem Jahr abgeschlossen wurde.

${ }^{6}$ Dieser Versuchung ist teilweise die Studie von P. Cox Miller, Dreams in Late Antiquity. Studies in the Imagination of a Culture, Princeton/New Jersey 1994, erlegen. S. dazu C. Walde, MH (= Museum Helveticum) 52 (1995), S. 259 f. Vgl. dagegen die umsichtigen Bemerkungen von H. Strasburger, Psychoanalyse und Alte Geschichte, in: ders., Studien zur Alten Geschichte II, hg. v. W. Schmitthenner/R. Zoepffel, Hildesheim, New York 1982, S. 1098-1110, bes. 1104 (Erstveröffentlichung). 
II.

Zunächst einige Vorbemerkungen zum Traum in der Antike generell und zur Abgrenzung des Themas: Träume finden sich seit Homer über die christliche Spätantike hinaus in fast allen literarischen Gattungen belegt, nicht selten auch auf ihre Bedeutung hin befragt. ${ }^{7}$ Es handelt sich dabei sowohl um gesprochene Traumbotschaften als auch um Bildfolgen und Symbole, die einer Deutung bedürfen. Allein die Liste der hiermit befaßten Denker, von Heraklit über Platon und Aristoteles bis zu Augustinus, nimmt sich beeindruckend aus: Gegenstand antiker Reflexion waren die Herkunft der Träume - von außen, d.h. von den Göttern oder von Dämonen bzw. Engeln, oder von innen, d.h. aus Körper oder Seele des Menschen selbst, sozusagen Tagesreste - sowie ihre Glaubwürdigkeit; außerdem hat man sich immer wieder um die Aufstellung von Kriterien bemüht, nach denen sich die gegebene Deutung erfüllen werde. Selbst wenn es vornehmere Formen der Divination gab und Aristoteles nichts vom Verlaß auf Traumdeutung hielt, Vertrauen in die Signifikanz der Träume ist in Zeugnissen quer durch alle Schichten, vom Herrscher bis zum Sklaven, bezeugt, und man hat nicht den Eindruck, daß hiermit jemand als $\delta \varepsilon \imath \sigma \_\delta \alpha i ́ \mu \omega v$, als abergläubisch, diskreditiert werden konnte. ${ }^{8}$ Hierbei beeindrucken besonders die zahlreichen, im Traum vorgenommenen Heilungen in den Heiligtümern von Asklepios, Sarapis und Isis. ${ }^{9}$

Deuten konnte jeder selbst, doch gab es auch professionelle Traumdeuter auf Märkten und bei Festen, innerhalb von Heiligtümern und unmittelbar vor ihnen; Herrscher umgaben sich mit ständig anwesenden Deutern. Dabei gilt es zu betonen, daß Traumdeutung in der Antike nicht das Ziel verfolgte, mit Hilfe der Traumbilder der Persönlichkeit des Träumenden, vor allem ihren grundlegenden Prägungen in der Vergangenheit, auf die Spur zu kommen. ${ }^{10}$ Das Ziel bestand vielmehr darin, mittels diverser

${ }^{7}$ Einen knappen Abriß mit den wichtigsten Fragen bieten J. Latacz, Funktionen des Traums in der antiken Literatur, in: T. Wagner-Simon/G. Benedetti (Hg.), Traum und Träumen, Göttingen 1984, S. 10-31, u. B. Manuwald, Traum und Traumdeutung in der griechischen Antike, in: R. Hiestand (Hg.), Traum und Träumen. Inhalt, Darstellung, Funktionen einer Lebenserfahrung in Mittelalter und Renaissance, Düsseldorf 1994, S. 15-42. Zahlreiche Traumtexte, jedoch mit unzureichender Kommentierung, bietet L. Hermes, Traum und Traumdeutung in der Antike, Zürich, Düsseldorf 1996.

${ }^{8}$ Das bekannte Zeugnis Theophrasts (Char. 16,11) karikiert auch nur das sofortige und ständige Aufsuchen eines Deuters, dazu Stewart, La religione (wie Anm. 2), S. 606, 610; G. Guidorizzi, Sogno e funzioni culturali, in: ders. (Hg.), Il sogno in Grecia, Bari 1988, S. VIIXXXVIII, hier XX.

${ }^{9} \mathrm{Vgl}$. hierzu die klassische Abhandlung von R. Herzog, Die Wunderheilungen von Epidauros. Ein Beitrag zur Geschichte der Medizin und der Religion (Philologus Suppl. 22/3), Leipzig 1931; zuletzt L. R. LiDonnici, Compositional Background of the Epidaurian 'Iámata', $\mathrm{AJPh}$ (= American Journal of Philology) 113 (1992), S. 25-41.

${ }^{10}$ Aus der Vielzahl der Stellungnahmen zum Verhältnis antiker zu moderner Traumdeutung sei verwiesen auf G. Steiner, The Historicity of Dreams (Two Questions to Freud), Salmagundi 61 (1983), S. 6-21, u. S. R. F. Price, The Future of Dreams. From Freud to Artemidorus, P\&P (= Past and Present) 113 (1986), S. 3-37. 
Analogieverfahren die gegebenen Informationen - dazu zählen die Traumerzählung selbst sowie Details aus der Biographie des Träumers, seinem Lebensumfeld etc. -, also Bilder und Worte der Traumwelt in die Wachwelt zu übertragen und herauszuarbeiten, welche exakte Bedeutung der Traum für einen bestimmten Träumenden haben wird. Nachvollziehen läßt sich dies am einzigen erhaltenen antiken Traumbuch, dem des Artemidor von Daldis aus dem 2. Jh. n.Chr., in dem eine Fülle von Deutungen für Personen mit unterschiedlichem Sozialstatus zusammengestellt ist. ${ }^{11}$

Was die Gestalt der überlieferten Träume angeht, so können wir eine Vielzahl konstanter Faktoren durch die ganze Antike hindurch festmachen, z.B. die Literarisierung homerischer Modelle, etwa des trügerischen Botschaftstraumes von Zeus an Agamemnon im zweiten Buch der Ilias und der Rede der Penelope von den zwei Toren, durch welche die Träume kommen, im 19. Buch der Odyssee. ${ }^{12}$ Deshalb bedarf es der Rechtfertigung des gewählten Zeitabschnitts:

(1) Religion und Erfahrungen des Individuums waren im Hellenismus teilweise von anderer Qualität als zuvor und danach. ${ }^{13}$

(2) Mit der hellenistischen Monarchie bildete sich ein neuer politischer, religiöser und auch mentaler Faktor heraus, der sich möglicherweise auch in den überlieferten Träumen und deren Interpretation niederschlug.

(3) Der besondere Kontakt mit Mesopotamien und Ägypten konnte neue Einflüsse auf den bisherigen griechischen Gebrauch bewirken.

(4) Für den Hellenismus liegt im Vergleich mit dem Klassischen Griechenland und mit der Römischen Kaiserzeit relativ reiches und vielfältiges Material vor. ${ }^{14}$

${ }^{11}$ Dazu L. H. Martin, Artemidorus. Dream Theory in Antiquity, SCent 8 (1991), S. 97-108, dort auch zu den geistesgeschichtlichen Voraussetzungen der Übertragung von Traumsymbolen in die Wachwelt; jetzt auch G. W. Bowersock, Fiction as History. Nero to Julian, Berkeley u.a. 1994, S. 77-98 („,The Reality of Dreams“). Daß sich aufgrund dieser Korrelation die gegebenen Traumdeutungen auch sozialgeschichtlich auswerten lassen, zeigt I. Hahn, Traumdeutung und gesellschaftliche Wirklichkeit. Artemidorus Daldianus als sozialgeschichtliche Quelle (Xenia 27), Konstanz 1992 (urspr. ungarisch, Budapest 1984).

${ }^{12}$ Zuletzt hierzu C. Brillante, Studi sulla rappresentazione del sogno nella Grecia antica, Palermo 1991, S. 32 ff., 166 f. mit Anm. 46, u. Cox Miller, Dreams (wie Anm. 6), S. 14 ff.

${ }^{13}$ Das $\mathrm{Ma}$ an tatsächlicher Veränderung bzw. Kontinuität der Polisreligion ist umstritten und in der Beurteilung oft abhängig vom zugrundegelegten Quellenmaterial bzw. dem Blickwinkel (Herrscher, Polis, Individuum); dazu mit der notwendigen Differenzierung R. Gordon, Fear of Freedom? Selective Continuity in Religion During the Hellenistic Period, Didaskalos 4 (1972), S. 48-60; A. Swiderek, La cité grecque et l'évolution de la mentalité religieuse dans les premiers temps de l'époque hellénistique, Eos 78 (1990), S. 259-272; bes. F. Graf, Bemerkungen zur bürgerlichen Religiosität im Zeitalter des Hellenismus, in: M. Wörrle/P. Zanker ( $\mathrm{Hg}$.), Stadtbild und Bürgerbild im Hellenismus (Vestigia 47), München 1995, S. 103-114, der zu dem Ergebnis gelangt: „Damit erweist sich die hellenistische Religion als traditioneller, als man oft gemeint hat" (S. 114). Wichtig erscheint vor allem der Hinweis auf die „Zweipoligkeit des einzelnen“, der durch die Traditionen der 'alten' Polis geprägt ist und aufgrund der tatsächlichen Ausweitung seines Aktionsraumes auf sich selbst zurückgeworfen ist und nach neuen Lösungen suchen muß. Zur Veränderung individueller Erfahrungen vgl. auch H.-J. Gehrke, Geschichte des Hellenismus (Oldenbourg Grundriß der Geschichte 1A), München 1990, S. 71 ff.

${ }^{14}$ Dies bedeutet aber auch, daß die Vergleichsmöglichkeiten mit anderen Epochen der griechisch-römischen Antike recht eingeschränkt sind. Denn aus den in hellenistischer Zeit erhal- 
III.

Im folgenden soll der von Träumen bestimmte Alltag des hellenistischen Menschen skizziert werden. ${ }^{15} \mathrm{Da} ß$ die erhaltenen Zeugnisse in engem Kontext mit Gottheiten oder deren Heiligtümern stehen, verwundert nicht, da die Rückbindung an eine höhere Autorität die Voraussetzung dafür war, daß aus einem Traum Konsequenzen gezogen wurden. Einige markante Beispiele aus unterschiedlichen sozialen Zusammenhängen und Quellengattungen mögen genügen.

Zunächst liegt eine Vielzahl von Weihungen vor, in deren Inschrift sich der dankbare Dedikant auf einen göttlichen Auftrag beruft. Im Falle von $\kappa \alpha \tau^{\prime}$ 'ov $\alpha \rho$ oder $\kappa \alpha \tau$ ' $\varepsilon v v ́ \pi v ı v$ ist das Medium der Vermittlung eindeutig; da aber auch andere Arten der göttlichen Kontaktaufnahme denkbar sind, läßt sich der Bezug zum Traum bei Formulierungen wie $\kappa \alpha \tau$ ' $\dot{\pi} \tau \imath \alpha \gamma \eta \dot{v}$,

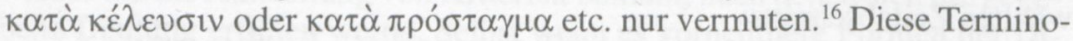
logie ist insofern bemerkenswert, als sie gerade in hellenistischer Zeit die Götter in der Diktion machtvoller Herrscher präsentiert. ${ }^{17}$ Die Analyse der in den Texten angesprochenen Götter ergibt nicht nur ein sehr weites Spektrum, sie stellt auch klar, daß man keineswegs immer an einen inkubatorischen Kontext oder an eine Heilung zu denken hat. Die Dedikationen sind aber nicht allein auf Traumgeheiß erfolgt - in den meisten Fällen wird dies gerade nicht vermerkt; deshalb ist die ausdrückliche Berufung auf die-

tenen Träumen auf Papyrus darf z.B. nicht geschlossen werden, daß zuvor etwas Derartiges nicht verzeichnet wurde.

${ }^{15} \mathrm{~N}$. Elias, Zum Begriff des Alltags, in: K. Hammerich/M. Klein (Hg.), Materialien zur Soziologie des Alltags, Opladen 1978, S. 22-29; H. Bausinger, Erlebte Geschichte - Wege zur Alltagshistorie, Saeculum 43 (1992), S. 95-107; L. Niethammer, Anmerkungen zur Alltagsgeschichte, in: K. Bergmann/R. Schörken (Hg.), Geschichte im Alltag - Alltag in der Geschichte, Düsseldorf 1982, S. 11-29.

${ }^{16} \mathrm{Zu}$ Material und Terminologie vgl. S. Newhall, Quid de somniis censuerint quoque modo eis usi sint antiqui quaeritur, Diss. Harvard (microfilm ed.) 1913; A. D. Nock, Studies in the Graeco-Roman Beliefs in the Empire, JHS (= Journal of Hellenic Studies) 45 (1925), S. 84101 [zit. nach Z. Stewart (Hg.), Artur Darby Nock. Essays on Religion and the Ancient World, London 1972, S. 33-48, 45 ff.]; J. S. Hanson, Dreams and Visions in the Graeco-Roman World and Early Christianity, in: ANRW (= Aufstieg und Niedergang der Römischen Welt) II 23/2, Berlin, New York 1980, S. 1395-1427; F. T. van Straten, Daikrates' Dream. A Votive Relief from Kos, and Some Other kat'onar Dedications, BABesch (= Bulletin Antieke Beschaving) 15 (1976), S. 1-38, bes. 21-27; M. L. Lazzarini, Iscrizioni votive greche, in: Scienze dell' antichità. Storia archéologia antropologia 3/4 (1989-90), S. 845-859, hier 852 ff.: „Non possiamo determinare con certezza come il fedele ricevesse o credesse di ricevere ordini o sollecitazioni. Certo è che la più antica forma di manifestazione divina sembra essere stata quella del sogno" (S. 853); Leuci, Terms (wie Anm. 4) passim.

${ }^{17}$ Hierzu Nock, Studies (wie Anm. 16), S. 47 f.; van Straten, Daikrates', ebd., S. 12 f., u. ders., Images of Gods and Men in a Changing Society. Self-identity in Hellenistic Religion, in: A. W. Bulloch et al. (Hg.), Images and Ideologies. Self-definition in the Hellenistic World (Hellenistic Culture and Society 12), Berkeley u.a. 1993, S. 248-264, hier 259. Wichtig dazu noch die Beobachtungen von M.-Th. Baslez, Recherches sur les conditions de pénétration et de diffusion des religions orientales à Délos, Paris 1977, S. 118 f., 289 ff., zur orientalischen

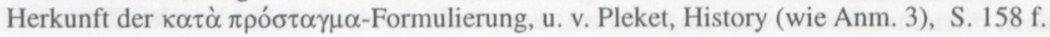


sen Zusammenhang ein auf der Folie der üblichen Polisreligion bedeutsamer Versuch, sich selbst mittels der Weihung als in einem besonderen Verhältnis zu einer Gottheit stehend darzustellen; die Schriftträger lassen darüber hinaus auch erkennen, daß die Dedikatoren kaum allein den unteren Schichten zuzuweisen sind.

Einen außergewöhnlichen Fall stellt die Stiftung eines ganzen Kultbezirks

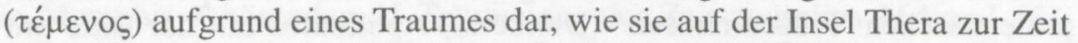
des dritten Ptolemäers (246-221 v.Chr.) für einen Artemidor aus Perge belegt ist: Dieser hat ein in den Fels gehauenes Ensemble von Altären, Reliefs mit Emblemen von Tieren und einem Selbstporträt mit Inschriften und wohl auch Statuen errichten lassen. Im Zentrum steht ein Homonoia-Altar, des-

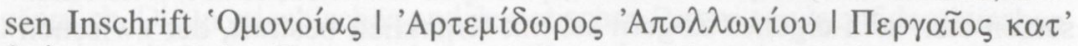

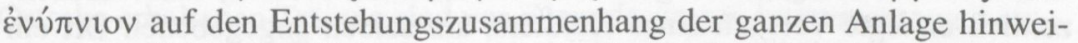
sen dürfte: ${ }^{18}$ Offenkundig hat Artemidor in einer nicht mehr rekonstruierbaren Situation zum Wohle der Homonoia auf Thera gewirkt und somit ein Verhältnis eigener Art auch zur Polis aufgebaut; mit dem Verweis auf die Trauminspiration konnte er seine Rolle legitimieren. ${ }^{19}$ Wie ernst man dies nahm, läßt sich daran ersehen, daß Artemidor Bürger von Thera wurde und bei den lokalen Arsinoeia einen Olivenkranz zuerkannt bekam, nach seinem Tod einen von Delphi approbierten Heroenkult erhielt und daß 'sein' heiliger Bezirk priesterlich weiterbetreut wurde. ${ }^{20}$ All diesen Dedikationsinschriften ist ein Merkmal gemeinsam: Der exakte Inhalt, Bilder und Worte der Träume wurden nicht für mitteilenswert erachtet, die Berufung auf Traum, Vision oder den Auftrag einer Gottheit reichte offenbar aus. ${ }^{21}$ Eine Möglichkeit

18 „Für Homonoia. Artemidor, Sohn des Apollonios, aus Perge. Auf Traumgeheiß““. IG XII ${ }^{3}$ Suppl. 1336. Vgl. Prosopographia Ptolemaica, bearb. v. W. Peremans et al., Löwen 1950 ff., VI 15188 (im folg. PP VI), dazu die Hinweise bei R. S. Bagnall, The Administration of the Ptolemaic Possessions Outside Egypt, Leiden 1976, S. 133 f. mit Anm. 64. Artemidor fand das Interesse einiger jüngst publizierter Studien: O. Palagia, Cult and Allegory. The Life Story of Artemidoros of Perge, in: J. Motyka Sanders (Hg.), Philolakon. Lakonian Studies in Honour of H. Catling, London 1992, S. 171-177, deren Argumente (173 f.) gegen die Interpretation als Stifterporträt nicht überzeugen; van Straten, Daikrates' (wie Anm. 16), S. 18, Anm. 260 (mit Abb. 24-28), u.a. mit dem Hinweis auf vergleichbare Stiftungen; bes. Graf, Bemerkungen (wie Anm. 13), S. 107-112 (in Anm. 36 die wichtigste Literatur), mit einer im Gegensatz zu Palagia konzisen Gesamtdeutung aller vorkommenden Gottheiten und Personifikationen im Hinblick auf die Polis Thera und die Biographie des Stifters. Lesenswert bleibt immer noch U. v. WilamowitzMoellendorff, Der Glaube der Hellenen, Berlin 1932, II, S. 387 ff.

${ }^{19}$ Graf, Bemerkungen (wie Anm. 13), S. 107 ff. mit Anm. 42. „It is interesting that a personification like Homonoia by this time had acquired sufficient body to appear in a dream", so van Straten, Daikrates'(wie Anm. 16), S. 18, dort Anm. 261, eine Zusammenstellung weiterer Traum-Personifikationen.

${ }^{20}$ IG XII ${ }^{3}$ Suppl. 1343, 1344 u. 1349. Das Ehrendekret mit der Bürgerrechtsverleihung ist verloren. Die permanente Wiederholung des Namens 'A $\rho \tau \varepsilon \mu i \delta \omega \rho \circ \varsigma$ wirkt ungemein penetrant.

${ }^{21}$ Dies hing sicher zum einen an den daraus resultierenden Mehrkosten für die Inschrift; zum anderen konnten mehr Details u.U. auch mehr Skepsis hervorrufen, etwa wenn ein Traum stark einem literarischen Muster verpflichtet war. Mit dem reinen Hinweis auf den Traumauftrag des Gottes blieb man dagegen unangreifbar. 
zur Nachprüfung, ob der behauptete Traum tatsächlich stattgefunden hatte, gab es nicht.

Die im folgenden besprochenen Papyruszeugnisse betreffen das ptolemäische Ägypten des 3. bzw. 2. Jh. und stehen in engem Kontakt mit der griechischen bzw. ägyptischen Ausprägung des Sarapis. ${ }^{22}$ In einem Brief vom 12. Februar 257 wendet sich ein gewisser Zoilos aus Aspendos an den ptolemäischen 'Finanzminister' (Dioiketes) Apollonios mit der Bitte um Hilfe: ${ }^{23} \mathrm{Ihm}$, einem eifrigen Verehrer des Sarapis, der auch fortwährend für das Wohl des Dioiketen und des Königs bete, sei der Gott mehrfach im Schlaf erschienen, damit er den ergangenen Auftrag ( $\chi \rho \eta \mu \alpha \tau \imath \sigma \mu o ́ s)$ dem Dioiketen kundmache $;{ }^{24}$ es sei ein $\tau \varepsilon \dot{\mu} \mu \varepsilon v o \zeta$ zu errichten, ein Priester einzusetzen etc.; sein eigenes Zögern sei mit Krankheit bestraft, der zwischenzeitlich erfolgte Versuch eines Knidiers, am selben angegebenen Ort ein Heiligtum zu errichten, vom Gott persönlich verhindert worden. Der Brief schließt mit der Aufforderung:

„Es verhält sich freilich wohl, Apollonios, daß du den vom Gott ergangenen Anweisungen Folge leistest, damit Sarapis dir gnädig ist und dich an Einfluß und Prestige beim König sowie an körperlichem Wohlergehen wachsen läßt."25

Dies ließe sich leicht als Versuch interpretieren, sich bei Apollonios unter Vorspiegelung religiöser Gefühle beliebt machen zu wollen, doch besteht keine Veranlassung, an deren Echtheit zu zweifeln. ${ }^{26}$ Die Wiederholung von Träumen, ebenso auch die Krankheit des Träumenden, sind übliche Vor-

${ }^{22} \mathrm{Zu}$ Sarapis zuletzt W. Huß, Der makedonische König und die ägyptischen Priester. Studien zur Geschichte des ptolemaiischen Ägypten (Historia-Einzelschriften 85), Stuttgart 1994, S. 58-68.

${ }^{23}$ P. Cair. Zen. 1,59034 (= PSI IV 435); zur Person PP VI 15758. Der Text ist mehrfach behandelt worden: vgl. U. Wilcken, Urkunden der Ptolemäerzeit I, Berlin 1927, S. 350, Anm. 1; F. Heichelheim/A. Storch, Zum Traumglauben und Traumverständnis in der Antike auf Grund urkundlicher Traumberichte aus der Ptolemäerzeit, Zentralblatt für Psychotherapie 4 (1931), S. 559-569, hier 559 f., Anm. 4; K. Latte, Rez. A. D. Nock, Conversion. The Old and the New in Religion from Alexander the Great to Augustine of Hippo, Oxford 1933, in: GGA (= Göttingische Gelehrte Anzeigen) 197 (1935), S. 114; H. Bell, Popular Religion in GraecoRoman Egypt, JEA (= Journal of Egyptian Archaeology) 34 (1948), S. 82-97, hier 94, dort auch Zustimmung zu dem im folgenden geäußerten Gedanken; del Corno, Contributi (wie Anm. 4), S. 113; Leuci, Terms, ebd., S. 48 f. Eine kommentierte Übersetzung bei N. Lewis, The Interpretation of Dreams and Portents, Toronto, Sarasota 1976, S. 45.

${ }^{24} \mathrm{Zu}$ dieser Art göttlicher Offenbarung vgl. Bell, Popular Religion (wie Anm. 23), S. 94 f.; Lazzarini, Iscrizioni (wie Anm. 16), S. 854 f. mit Anm. 104-106.

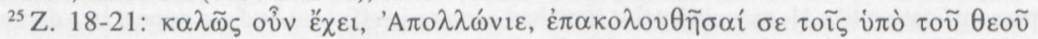

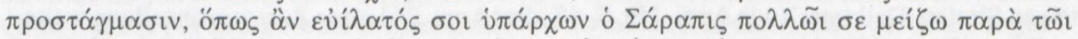

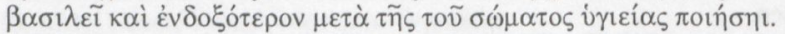

${ }^{26}$ Als weiteres Beispiel sei auf den Brief P. Cair. Zen. 3,59426,6 f. (=Sel. Pap. I 91; zw. 260 u. 250 v.Chr.) verwiesen, in dem Dromon (zur Person: P. W. Pestman et al., A Guide to the Zenon Archive [P.L. Bat. 21], 2 Bde., Leiden 1981, S. 321) den Zenon bittet, eine Kotyle attischen Honigs zu besorgen, „denn ich habe Bedarf daran für die Augen gemäß dem Erlaß

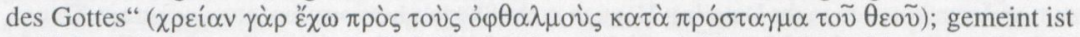
wohl Sarapis (vorausgesetzt, der Brief ist in Memphis geschrieben) oder Asklepios; vgl. noch Bell, Popular Religion (wie Anm. 23), S. 95. 
kommnisse: Nach Artemidors Traumbuch (4, 27, 261, 18-21 ed. Pack) machen sie die Traumbotschaft besonders dringlich; zwar wird man dem Träumenden eine intensive Beschäftigung mit seinem Bauvorhaben in Rechnung stellen, doch spricht der Befund an sich nicht unbedingt für eine literarische Stilisierung.

Am meisten beeindrucken die zum sogenannten 'Archiv des Ptolemaios' in Memphis gehörenden 35 Träume aus der Zeit zwischen 164 und 151 v.Chr., die sich zwischen Urkunden, Rechnungen und literarischen Texten befanden. Sie wurden bereits in der ersten Hälfte des vergangenen Jahrhunderts gefunden, an verschiedene Bibliotheken verkauft und sind erst durch Ulrich Wilckens meisterliche Ausgabe aus dem Jahre 1927 geschlossen zugänglich. Die in griechischen und demotischen Papyri sowie auf demotischen Ostraka erhaltenen Träume sind vor allem deswegen interessant, weil sie mit einer Ausnahme ${ }^{27}$ - zu den ganz wenigen bislang bekannten Träumen aus griechisch-römischer Zeit gehören, die keine Anzeichen der üblichen literarischen Ausgestaltung aufweisen, also direkt aufgezeichnet wurden und somit für ein hohes $\mathrm{Maß}$ an Authentizität stehen; ${ }^{28}$ man wird davon ausgehen müssen, daß auch sie festen Mustern folgten, doch entsteht auf der Folie der literarischen Träume der Eindruck von Unmittelbarkeit, zumal die Traumsequenzen für sich stehen und weder eine Deutung noch ein Indiz für die Erfüllung des Traumes beigegeben ist. Deshalb kann man versuchen, die Traumszenerie und -symbolik mit den sonstigen Informationen über das sehr bewegte Leben der Protagonisten in Beziehung zu setzen.

${ }^{27}$ Diese betrifft den 'Traum des Nektanebo' (UPZ 81 u. L. Koenen, The Dream of Nektanebos, in: D. Hobson/K. McNamee [Hg.], Classical Studies Presented to W. H. Willis [BASP 22], New York 1985, S. 171-194), den offenkundig Apollonios, der Bruder des Ptolemaios, abgeschrieben hat. Näheres bei A. L. Oppenheim, The Interpretation of Dreams in the Ancient Near East, with a Translation of an Assyrian Dream-Book, TAPhA (= Transactions and Proceedings of the American Philological Association) 46 (1956), S. 179-355, hier 196 mit Nr. 23; D. J. Thompson, Memphis under the Ptolemies, Princeton 1988, S. 262 f.; Huß, Der makedonische König (wie Anm. 22), S. 133-137.

${ }^{28}$ Wilcken, Urkunden (wie Anm. 23), als Nr. 2-105; die Träume enthaltenden Dokumente sind die Nummern 77-81, dazu Andeutungen in 68-70 (im folg. abgekürzt UPZ). Nicht mitgerechnet sind die Traumthemen in UPZ 80. Eine Beschreibung des Materials gibt L. Delekat, Katoche, Hierodulie und Adoptionsfreilassung (Münchener Beiträge zur Papyrusforschung und antiken Rechtsgeschichte 47), München 1964, S. 140 (diese Studie erfuhr der L'Année Philologique zufolge mindestens 17 Besprechungen, die sich jedoch fast ausschließlich auf die rechtshistorische Relevanz und nicht auf die behandelten Träume bezogen). Zu den demotischen Texten s. G. Botti, Testi demotici 1, Florenz 1941, S. 10-19. (Nr. 2 u. 3), die italienische Übersetzung ist auch zugänglich bei Delekat (wie oben), S. 151, 155, Anm. 2; eine Neulesung bieten E. Bresciani/ E. Bedini/L. Paolini/F. Silvano, Una rilettura dei Pap. dem. Bologna 3173 e 3171, EVO (= Egitto e vicino oriente) 1 (1978), S. 95-104, die allerdings aufgrund der demotischen Namen die Zugehörigkeit zum UPZ-Corpus anzweifeln; eine englische Übersetzung bei Lewis, The Interpretation (wie Anm. 23), S. 47-52. Zum gesamten Kontext vgl. Heichelheim/ Storch, Traumglauben (wie Anm. 23); del Corno, Contributi (wie Anm. 4), S. 111 ff.; N. Lewis, Greeks in Ptolemaic Egypt. Case Studies in the Social History of the Hellenistic World, Oxford 1986, S. 69-87, u. Thompson, Memphis (wie Anm. 27), S. 212-265; Leuci, Terms (wie Anm. 4), S. 49-55. Der Versuch einer konzisen Deutung aller Träume vor dem Hintergrund der sonstigen Dokumente ist noch nicht unternommen worden, vgl. immerhin die methodischen Leitlinien bei Delekat (wie oben), S. 140. 
Besagter Ptolemaios befand sich in dem zum memphitischen Serapeion

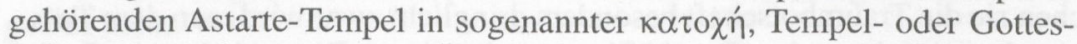
haft, und war dort, trotz mancher ethnischer Konflikte, als Makedone sehr angesehen. Wahrscheinlich kam er als Asylsuchender in das Heiligtum und verpflichtete sich aus unbekannten Gründen zum Dienst für den Gott, was in aller Regel durch einen Traum bestätigt wurde. ${ }^{29}$ Die Träume stammen nicht allein von Ptolemaios selbst, sondern auch von weiteren Personen aus seinem Tempel-Umfeld: von seinem jüngsten Bruder Apollonios, von zwei mit beiden eng befreundeten Mädchen ägyptischer Abkunft, den Zwillingen Tagês und Taus und von einem weiteren Ägypter namens Nektembes; dessen Träume waren von den vier zuvor genannten Personen möglicherweise in Auftrag gegeben, d.h. als Fragen in eine Inkubation hineingenommen und von Ptolemaios aufgezeichnet worden. ${ }^{30}$ Das Lebensumfeld all dieser Personen im Tempelbereich ist zwar stark von ausgeprägten Handelsaktivitäten gekennzeichnet, doch scheint die religiöse Komponente immer durch; Träume sind dabei quasi selbstverständlich.

Drei Träume mögen genügen, um Duktus und Probleme der Interpretation aufzuzeigen. ${ }^{31}$ Der Inhalt eines demotischen Petersburger Ostrakon wird wie folgt resümiert: ${ }^{32}$

„Die Träumende [sc. eine der Zwillingsschwestern] sieht sich in Memphis; man sagt ihr, daß ihre Mutter Nephoris von der steigenden Überschwemmung bedroht sei. Sie schwimmt hinüber und rettet sie in das Anubieion [...]“

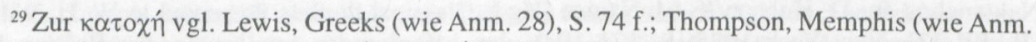

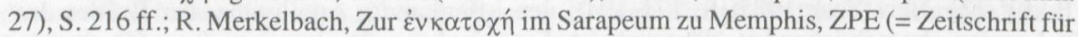
Papyrologie und Epigraphik) 103 (1994), S. 293-96. Auf die Frage des Tempelasyls in hellenistischer Zeit kann hier nicht weiter eingegangen werden, vgl. dazu F. v. Woess, Das Asylwesen Ägyptens in der Ptolemäerzeit und die spätere Entwicklung, München 1923; H. Heinen, Ägyptische Tierkulte und ihre hellenistischen Protektoren. Überlegungen zum Asylieverfahren SB III 6154 (= IG Fay. III 135) aus dem Jahre 69 v.Chr., in: M. Minas/J. Zeidler (Hg.), Aspekte spätägyptischer Kultur. FS E. Winter zum 65. Geburtstag, Mainz 1994, S. 157-168; jetzt auch K. J. Rigsby, Asylia. Territorial Inviolability in the Hellenistic World, The University of California Press 1996.

${ }^{30} \mathrm{Da} \beta$ eine Person für die andere träumen konnte, war gängige Praxis. Zur Inkubation in ägyptischen Tempeln und zur damit verbundenen Traumdeutung vgl. J. Ray, The Archive of Hor, London 1976, S. 130-136, der in diesem Band die zweite bedeutende, aus der gleichen Zeit stammende Gruppe von Träumen in demotischer Sprache ediert hat; außerdem Thompson, Memphis (wie Anm. 27), S. 208 ff. Einen weiteren, in der Interpretation problematischen demotischen Traumtext aus der Zeit des zweiten Ptolemäers behandelt J. D. Ray, An Agricultural Dream. Ostracon BM 5671, in: J. Baines et al. (Hg.), Pyramid and Other Essays Presented to I. E. S. Edwards, London 1988, S. 183 mit Abb. 38, der dazu noch auf das inhaltlich u.U. verwandte und bis auf wenige Tage zeitgleiche O. Brooklyn $37.1821 \mathrm{E}$ verweist.

${ }^{31}$ Versuche wurden unternommen von Heichelheim/Storch, Traumglauben (wie Anm. 23), S. $566 \mathrm{ff}$., oft spekulativ und zu sehr psychologisierend, und von Delekat, Katoche (wie Anm. 28), S. $136 \mathrm{ff}$., der das Verständnis der beiden überaus komplexen, hier nicht behandelten Traumsequenzen von UPZ 78 entscheidend gefördert hat.

${ }^{32}$ OPetersburg 1129, zit. nach Wilcken, Urkunden (wie Anm. 23), S. 351, bzw. Heichelheim/ Storch, Traumglauben (wie Anm. 23), S. 563 (Nr. 2), 566, dort auch die gegebenen Interpretationsalternativen. 
Eine Deutung des Traumes hätte zunächst den Familienhorizont zu berücksichtigen: Anlaß zur Flucht der Zwillinge in den Tempel war die Bedrohung durch die Mutter und der Tod des Vaters durch den Liebhaber der Mutter. Der Traum könnte dann besagen, daß zwischenzeitlich eine Versöhnung von Mutter und Tochter stattgefunden hat; psychologisierend wurde er jedoch auch in dem Sinne gedeutet, daß die Tochter die Mutter in Gefahr sehen wolle und sich ihr Haß somit zur Selbstrechtfertigung in ihr Gegenteil verkehre. $^{33}$

Ein weiteres Beispiel:

„Ptolemaios' Traum am 15. Pachon [14.6.161]. Es traten heran zu mir zwei
Männer und sagten zu mir: 'Ptolemaios, nimm das Kupfergeld für das Blut.'
Sie zählten mir Kupfergeld hundert Drachmen hin und der Tagês, der Zwil-
lingsschwester, Kupfergeld, in Stateren geprägt, einen vollen Beutel. Sie sag-
ten zu ihr: 'Siehe, das Kupfergeld für das Blut.' Ich sagte zu ihnen: 'Sie hat
mehr Kupfergeld als ich bekommen.' '“34

Eine Deutung mit Hilfe von Artemidors Traumbuch führt nicht weit: Kupfergeld steht nach Artemidor $(2,58)$ für Mißstimmung und Beleidigungen, Blut für Geld selbst $(1,33)$. An persönlichem Hintergrund wissen wir, daß Ptolemaios den Zwillingen als Freund ihres verstorbenen Vaters im Rechtsstreit mit der Mutter half; das Blut weist möglicherweise auf den von der Mutter mitverschuldeten Tod des Vaters; und daß nur Tagês Geld erhält, deutet auf die auch sonst aus den Träumen ersichtliche besondere Aufmerksamkeit seitens des Ptolemaios hin; schließlich ist die Klage über die finanzielle Benachteiligung - man hat zu fragen, ob ihm überhaupt etwas zusteht - eventuell Ausdruck seiner Enttäuschung darüber, daß Tagês, die zudem von seinem Bruder Apollonios ein Kind erwartet, ihm sein zweifellos beachtliches Engagement nicht genügend honoriert hat. ${ }^{35}$

Der folgende Traum entstammt einer Serie, die Nektembes für Ptolemaios erträumte, und steht im gleichen Kontext wie das vorherige Beispiel:

„[...] Ich glaubte, eine Taube [zu sehen], die war aus meiner Hand entflohen, und ich laufe ihr nach, indem ich sagte: 'Die will ich sicher nicht entfliehen

\footnotetext{
${ }^{33}$ So Heichelheim/Storch, ebd., S. 566. Der Hintergrund ist in UPZ 18 u.19 ausgeführt.

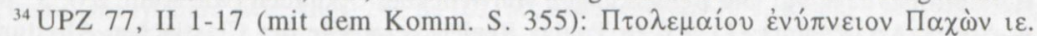

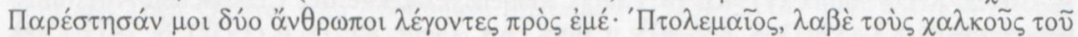

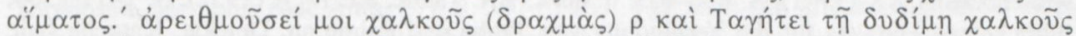

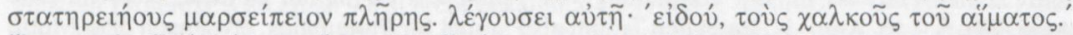

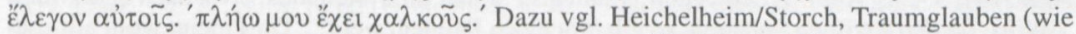
Anm. 23), S. 563 (Nr. 7), 567. In jeder Hinsicht unzutreffend ist die Wertung von Thompson, Memphis (wie Anm. 27), S. 225: „Although such dreams may tell more of fantasy than of real life, in recording them Ptolemaios shows the same troubled psychology as is revealed elsewhere - in his obsession with his accounts which were worked and reworked with a neurotic application unmatched by accuracy. "Dahinter steht u.a. die verbreitete Tendenz, antike Personen, die viele Träume aufgezeichnet haben (etwa Aelius Aristides), aufgrund dieses Zusammenhanges als neurotisch zu bezeichnen.

${ }^{35}$ Zur Charakterisierung der anderen Zwillingsschwester vgl. Delekat, Katoche (wie Anm. 28), S. 151 f.; Mutmaßungen über das nicht allein väterliche Interesse an den Zwillingen seitens des Ptolemaios bei Thompson, Memphis (wie Anm. 27), S. 224 f.
} 
lassen.' Ich greife sie und lege sie in meine linke Hand und ich hielt sie fest und [nahm] einen Palmenzweig, damit sie nicht entfliehe. “"36

Sucht man in Artemidors Traumbuch nach der Bedeutung der einzelnen Elemente, so stehen nach Artemidor Haustauben im Gegensatz zu Ringeltauben für Frauen, die ihr Haus unter Kontrolle haben und ordentlich sind; ,,sie bedeuten auch, weil sie der Aphrodite heilig sind, die Liebe und Lust an den Geschäften und sind günstig für Freundschaft, gemeinsame Unternehmungen und alle Verbindungen $[\ldots]^{‘ 37}$ Die linke Hand steht für die Bewahrung von bereits Erworbenem, ebenso für das weibliche Geschlecht $(1,42)$. Die Palme wird von Artemidor nur in Form eines Kranzes aus den Zweigen erwähnt (1,77): Dieser bedeutet Heirat mit einer freigeborenen Frau, langes Leben der Kinder, Verheißung eines Sohnes, dazu auch Reichtum für Arme, Freiheit für Sklaven - und sie bringen Verborgenes ans Licht. ${ }^{38}$ Hier läßt sich die systematisierte Traumsymbolik zwar anwenden, doch liegt der Zusammenhang wohl auch so auf der Hand: Ptolemaios möchte nicht, daß der betreffende Zwilling ihm abhanden kommt, entweder emotional oder durch tatsächliches Verlassen des Tempelbezirkes. ${ }^{39}$

Die drei behandelten Träume sind im Gegensatz zu den bisherigen Beispielen dadurch gekennzeichnet, daß in ihnen von Göttern nicht explizit die Rede ist,$^{40}$ wenngleich ein inkubatorischer Kontext gegeben sein dürfte. Die Träumenden sehen sich selbst wie in der Wachwelt in einer Abfolge von Handlungen agieren und interagieren. Nach Artemidors strenger, aber im Einzelfall kaum verifizierbarer Definition $(1,1 \mathrm{f}$.) wären dies auch keine zu

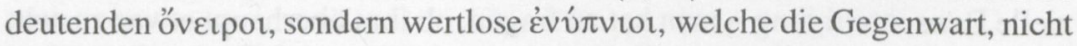

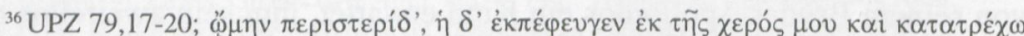

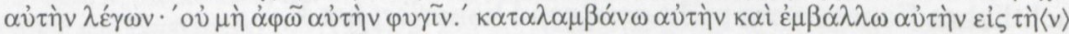

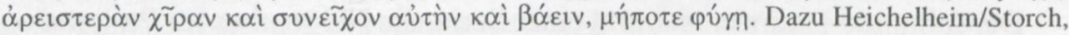
Traumglauben (wie Anm. 23), S. 565 (Nr. 12), 568 f.

${ }^{37}$ Artemidor, 2,20,137,14-18. Näher an der erwähnten Traumszenerie sind jüdische Deutungen zur Taube, vgl. A. Kristianpoller, Traum und Traumdeutung (Monumenta Talmudica IV, II: Aberglauben, Heft 1), Wien, Berlin 1923 (ND Darmstadt 1972), S. 41. f. (Nr. 123) u. 59 (Nr. 195).

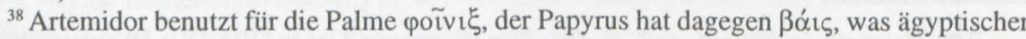
Herkunft ist, dazu vgl. die Bemerkungen von Wilcken, Urkunden (wie Anm. 23), S. 368; A. Steier, Art. Phoinix (1), RE (= Paulys Realencyclopädie der Classischen Altertumswissenschaft) XX (1941), S. 386-403, u. ders., Art. Taube, RE IV A (1932), S. 2479-2500, führt keinen Hinweis für die Verbindung von Taube und Palme an. Delekat, Katoche (wie Anm. 28), S. 146, Anm. 1, deutet den Traum als Parabel für Ptolemaios, etwa nach dem Motto „'Willst du den Zwilling behalten, so gib ihm einen Palmenzweig'“': Dies meint wohl am ehesten einen Palmzweig als besondere Auszeichnung, doch ist die Verbindung von Palmzweig und Siegespreis erst kaiserzeitlich belegt.

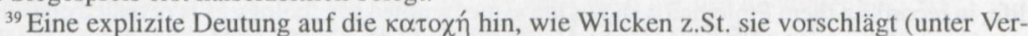

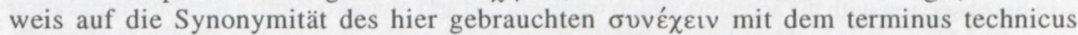
$\kappa \propto \tau \varepsilon ́ \chi \varepsilon \sigma \theta \alpha \iota)$, führt zu weit und trifft auch nicht den Sinn des Traumes. Delekats Vermutung, Katoche (wie Anm. 28), Nektembes könnte auch den Flug der Tauben gedeutet haben, ist willkürlich.

${ }^{40}$ In den Träumen ist mehrfach vom Sarapistempel die Rede, allein in UPZ 77, II 22-32 agiert der Gott Ammon als Geburtshelfer, auch von einer Kuh und einem Stier wird gesprochen, was nach Thompson, Memphis (wie Anm. 27), S. 195, durch die lokale Vorgabe des Apiskultes bedingt ist. 
die Zukunft anzeigen und als Tagesreste bezeichnet werden können; diese Unterscheidung ist bereits bei Aristoteles belegt, ${ }^{41}$ sie steht aber vor allem für den Spielraum des professionellen Deuters, einen Traum deuten oder aber die Deutung ablehnen zu können. $D a \beta$ die Träume aber aufgezeichnet wurden, spricht aus der Sicht der Träumenden für ihre Signifikanz, die zweifellos auch bei den Symbolträumen mit einer göttlichen Herkunft verbunden war.

Diese Träume verdeutlichen auch, daß die Anwendung eines Traumdeutungsbuches nur bedingt hilfreich ist, wenn sich die persönliche Situation des Träumenden nicht angemessen berücksichtigen läßt - genau dies ist heute nicht mehr möglich; zwar hat man davon auszugehen, daß Artemidor oder einem anderen Deuter in jedem Fall eine Deutung gelungen wäre, doch läßt sich die genaue Vorgehensweise trotz der zahlreichen praktischen Hinweise in seinem Buch nicht rekonstruieren. ${ }^{42}$ Diese grundlegende Inkonsistenz ist deshalb zu betonen, weil man mit der möglichen Andersartigkeit der Traumdeutung im rein ägyptischen Milieu argumentieren könnte; denn die Gesellschaft im memphitischen Serapeion setzte sich aus Griechen und Ägyptern zusammen, wie denn auch Träume des Apollonios in demotischer Sprache aufgezeichnet sind und ein Traum aus dem 'Archiv' innerhalb des griechischen Haupttextes griechische Transkriptionen offenkundig ägyptischer Worte aufweist. ${ }^{43} \mathrm{Zu}$ betonen ist aber, daß das einzige erhaltene Traumbuch in demotischer Sprache der Vorgehensweise des zeitgleichen Artemidor ähnelt, sieht man von spezifisch ägyptischen Traumthemen wie den Krokodilen oder dem Bier ab. ${ }^{44}$ Das erhaltene hieratische Traumbuch aus dem 13. Jh. v.Chr. hilft hier auch nicht weiter, zumal in pharaonischer Tradition di-

${ }^{41} \mathrm{Zu}$ den verschiedenen Deutungsmustern vgl. Cox Miller, Dreams (wie Anm. 6), S. 42 f.; P. J. van der Eijk, Aristoteles. De insomniis. De divinatione per somnum (Aristoteles. Werke in deutscher Übersetzung 14/III), Berlin 1994, S. 52 ff.

${ }^{42}$ Es ist aufgrund der Quellenlage fast unmöglich, eine generelle Aussage darüber zu treffen, wie zeitbezogen Traumdeutungsbücher waren: Artemidor hat zweifellos sehr viel älteres Material eingearbeitet (gegen das er z.T. auch polemisiert), weist aber keine über die eigene Zeit hinausgehenden historischen Bezüge auf.

${ }^{43}$ Die Autorschaft des Apollonios für die demotischen Texte lehnen Bresciani/Bedini/Paolini/

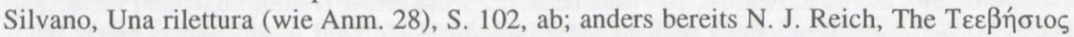
vioi and Their Quarrel with Apollonios, Mizraim 1 (1933), S. 147-177, bes. 156 ff.; bes. Thompson, Memphis (wie Anm. 27), S. 247 f., 263. Vgl. auch den griechischen Brief eines anderen Ptolemaios an einen sonst nicht bekannten (Ägypter?) Achilles (P. Goodsp. $3=$ Epist. priv. graec. Nr. 21, S. 30 f., ed. Witkowski; 3. Jh. v.Chr.), in dem der (verlorene) Inhalt eines Traumes, hier ó $\rho \alpha \mu \alpha$ genannt, in demotischer Sprache mitgeteilt wurde - weil in dieser Sprache dem anderen ein Traumbuch verfügbar war oder ,perhaps suggesting literacy in both languages", so Thompson (s. oben), S. 264? Dazu Wilcken, Urkunden (wie Anm. 23), S. 366 f. mit Anm. 3; Bell, Popular Religion (wie Anm. 23), S. 95 f.; del Corno, Contributi (wie Anm. 4), S. 112 .

${ }^{44}$ Das demotische Traumbuch edierte A. Volten, Demotische Traumdeutung (Pap. Carlsberg XIII und XIV verso), Kopenhagen 1942. Zu den hier angesprochenen Problemen vgl. del Corno, Contributi (wie Anm. 4), S. 110, 113 f.; Delekat, Katoche (wie Anm. 28), S. 136 ff.: „Es ist überhaupt nicht leicht zu sehen, wie anhand der in diesen Sammlungen zusammengestellten Traummotive jemand einen Traum zu deuten vermochte" (S. 138). 
rekte Traumgesichte, d.h. solche nicht strikt allegorischer Art, die Elite und nicht den Durchschnittsmenschen betrafen. ${ }^{45}$

$\mathrm{Zu}$ fragen hat man nach den Deutern: Inschriften weisen darauf hin, daß

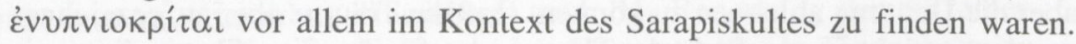
Aus Delos kennen wir eine Reihe von Belegen, ${ }^{46}$ besonders anschaulich ist jedoch das von Otto Rubensohn im Jahre 1900 erstmals richtig gedeutete Aushängeschild eines Traumdeuters, ebenfalls aus Memphis vom Ende des

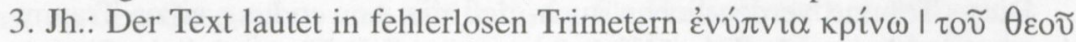

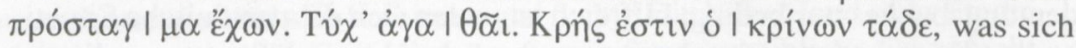
trotz des aufgemalten Apis-Stieres auch auf Träume anderer Götter beziehen konnte. ${ }^{47}$ Offenbar wurde im großen Areal des Serapeions eine derartige Aktivität seitens der Priester toleriert; bei einem Kreter, der sich ohne Namensnennung anpreist, dürfte man den gängigen Sprichwörtern zufolge vielleicht nicht die beste Reputation erwarten, doch ist davon auszugehen, daß mit dem Hinweis etwas Positives, vielleicht besondere Kompetenz, verbunden war; dazu findet sich noch der Hinweis auf göttliche Autorisierung in Form eines $\pi \rho o_{\sigma} \sigma \alpha \gamma \mu \alpha{ }^{48}$

Über die Methode der Deuter in hellenistischer Zeit wissen wir so gut wie nichts; Artemidor nennt zwar eine Reihe von Traumbuch-Autoren, auch klangvolle Namen wie Demetrios von Phaleron oder Straton von Lampsakos, doch sagen Werktitel und Umfangsangaben nichts über die Theorie und die praktizierten Deutungsmethoden aus. ${ }^{49}$ Relevant sind allenfalls noch theo-

${ }^{45}$ Zur sonstigen ägyptischen Traumdeutung vgl. K. Zibelius-Chen, Kategorien und Rolle des Traumes in Ägypten, SAK (= Studien zur altägyptischen Kultur) 15 (1988), S. 277-293; Texte, u.a. aus dem hieratischen Traumbuch, finden sich abgedruckt bei S. Sauneron, Les songes et leur interprétation dans l'Égypte Ancienne, in: Les songes et leur interprétation, Paris 1959, S. 17-61; dazu noch S. Israelitt-Groll, A Ramesside Grammar Book of a Technical Language of Dream Interpretation, in: dies. (Hg.), Pharaonic Egypt, the Bible and Christianity, Jerusalem 1985, S. 71-118. Bemerkenswert ist noch der Traum der Taimhotep, Frau des memphitischen Hohenpriesters Psenptais III. aus der Zeit Ptolemaios' XIII.: Imhotep-Asklepios verspricht im Traum gegen Bauarbeiten in seinem Tempel die Geburt des ersehnten Sohnes, dazu Thompson, Memphis (wie Anm. 27), S. 140 mit Belegen und Literatur.

${ }^{46}$ Siehe Anm. 51. Belege bei Wilcken, Urkunden (wie Anm. 23), S. 48, 391, der den UPZ 84, 79 genannten Deuter zu Unrecht unter das Tempelpersonal zählt; auch Delekat, Katoche (wie Anm. 28), S. 142 f., Anm. 6, zu Ptolemaios, der wohl selbst Träume zu deuten vermochte (UPZ 71, 2-5), sich dazu jedoch auch an andere wandte (UPZ 78, 1 u. 42), anders hierzu noch Heichelheim/Storch, Traumglauben (wie Anm. 23), S. 561. Außerdem noch Lazzarini, Iscrizioni (wie Anm. 16), S. 854 f.

${ }^{47}$ SB I 685: „Ich deute Träume in der Gottheit Auftrag. Sei Tyche hold. Ein Kreter ist es, der solches deutet.“ Dazu O. Rubensohn, Das Aushängeschild eines Traumdeuters, in: FS J. Vahlen, Berlin 1900, S. 3-15; Wilcken, Urkunden (wie Anm. 23), S. 13, 32; del Corno, Contributi (wie Anm. 4), S. 113 f., dessen Wertung der Deutung des Kreters in einem Sarapisheiligtum als Zeichen der 'Fusion' erst noch in einen umfassenderen Kontext zu stellen wäre; Thompson, Memphis (wie Anm. 27), S. 87, 225, 264 f. (mit Abb. VII).

${ }^{48}$ Hierzu Rubensohn, Aushängeschild (wie Anm. 47), S. 6 ff. Zur göttlichen Autorisierung auch A. D. Nock, A diis electa. A Chapter in the Religious History of the Third Century, HThR (= Harvard Theological Review) 23 (1930), S. 251-274 [zit. nach Stewart, Nock (wie Anm. 16), S. 252-270, hier 255 f.].

${ }^{49}$ Die griechischen Zeugnisse bei D. del Corno, Graecorum de re onirocritica scriptorum reliquiae, Mailand 1969 (dazu R. A. Pack, Gnomon 42 [1970], S. 833 f.; A. H. M. Kessels, 
retische Überlegungen zur Verwendung von Träumen in der Medizin, die von Herophilos, dem Arzt in Alexandria, aus dem 3. Jh. v.Chr. stammen. ${ }^{50}$

Noch ein Wort zur Terminologie: Der Befund in den Inschriften und Papyri zeigt, daß vom 4. bis zum 1. Jh. üblicherweise ع́vv́ $\pi v$ ıov verwendet wurde, danach eher öveıрo૬ oder öv $\alpha \rho$; der Traumdeuter der hellenistischen Zeit

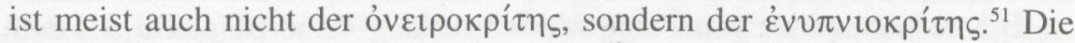
Dichter hingegen gebrauchen bevorzugt öveipos, auch óv $\alpha \rho$, und stehen damit sicher in guter literarischer Tradition. ${ }^{52}$ Eine mögliche Erklärung für

Mnemosyne 5 [1972], S. 202-205); außerdem B. Büchsenschütz, Traum und Traumdeutung im Alterthume, Berlin 1868, S. 45 ff.; Susemihl, Geschichte (wie Anm. 1), Bd. 1, S. 869 ff., 875; T. Hopfner, Art. Traumdeutung, RE VI A (1937), S. 2233-2245, hier 2238 ff.; Nilsson, Geschichte (wie Anm. 2), S. 230 f.; M. A. Vinagre Lobo, Etapas de la literatura onirocrítica según los testimonios de Artemidoro Daldiano, Habis 22 (1992), S. 297-312. Ob der aus dem nach bisherigen Kenntnisstand völligen Fehlen von Traumdeutungsliteratur auf Papyrus gezogene Schluß auf deren geringe Verbreitung berechtigt ist (so G. Guidorizzi, L'interpretazione dei sogni nel mondo tardoantico, in: T. Gregory [Hg.], I sogni nel Medioevo, Rom 1985, S. 149-170, hier 158), scheint fraglich zu sein, zumal man davon ausgehen kann, daß derartige Literatur, mit der ein sehr großes Wissen verbunden war und die in einem kommentierten Handexemplar auch praktische Notizen oder 'Erfahrungswerte' beinhalten konnte, im Zweifelsfalle eher versteckt oder vernichtet als zurückgelassen wurde.

${ }^{50}$ Vgl. P. H. Schrijvers, La classification des rêves selon Hérophile, Mnemosyne 30 (1977), S. 13-27; H. v. Staden, Herophilus. The Art of Medicine in Early Alexandria, Cambridge 1989 , S. 306-310 mit den Texten 226a-d. Zur Person G. Weber, Dichtung und höfische Gesellschaft. Die Rezeption von Zeitgeschichte am Hof der ersten drei Ptolemäer (Hermes Einzelschriften 62), Stuttgart 1993, S. 146 mit Anm. 4.

${ }^{51}$ Van Straten, Daikrates' (wie Anm. 16), S. 12 f., verzeichnet nur sehr wenige Ausnahmen, verweist allerdings auf eine Reihe nicht datierter Inschriften; Lazzarini, Iscrizioni (wie Anm. 16),

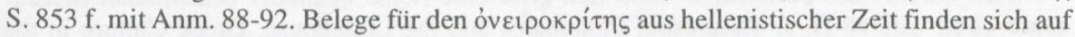
Delos, etwa ID 2072, 2073, 2105, 2106, 2110, 2120 und 2151, dazu Stewart, La religione (wie Anm. 2), S. 613.

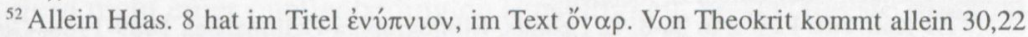
( $\dot{v} v \dot{\pi \nu} \alpha)$ in Frage, alle sonstigen Belege entstammen den unechten Gedichten $(9,16 ; 20,5)$, bemerkenswert ist 21,24 ff., nicht nur wegen der literarischen Gestaltung mittels eines Traumes, sondern auch wegen der geäußerten Ansichten 'einfacher Fischer' ( $32 \mathrm{f}$.: „Denn wenn auch nach dem Verstand ich sie deute, so ist doch der beste Deuter von Träumen wohl der, der es lernt vom eigenen Verstande“; 64: „das Gesicht war nichts anderes als Täuschung“) und der

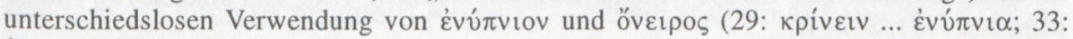

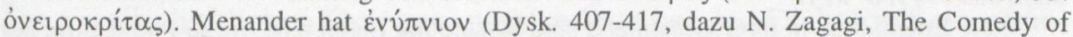
Menander. Convention, Variation and Originality, London 1994, S. 160 ff.). Bion, Epitaph.

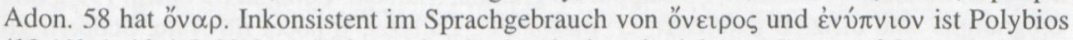
$(12,12 \mathrm{~b}, 1 ; 12,4,5 ; 33,21,1-2)$, besonders in der einzigen berichteten Traumerfahrung in $10,4,5$ ff. (Bewerbung der Scipionen um das Ädilenamt; zum weiteren Kontext vgl. F. Loretto, Träume und Traumglaube in den Geschichtswerken der Griechen und Römer, Diss. [masch.] Graz 1956, S. 159 f., Anm. 3.; C. J. Classen, Gottmenschentum in der römischen Republik, Gymnasium 70 [1963], S. 312-338, 318 f.; G. Maurach, Africanus Maior und die Glaubwürdigkeit des 'Somnium Scipionis', Hermes 92 [1964], S. 299-313, hier 301 f.; F. W. Walbank, The Scipionic Legend, PCPhS [= Proceedings of the Cambrigde Philological Society] 13 [1967], S. 54-69; H. H. Scullard, Scipio Africanus. Soldier and Politician, London 1970, S. 18-23; D. Potter, Prophets and Emperors. Human and Divine Authority from Augustus to Theodosius, Cambridge/Mass., London 1994, S. 147 f.), während in 10,11,7 das Traumgeschehen - Poseidon erscheint dem Scipio Africanus vor Karthago - eher umschrieben wird. Dafür, daß im literarischen Gebrauch eher övعıрoఢ üblich war, spricht auch die einmalige Verwendung des Wortes in UPZ 81, III 11 (siehe Anm. 27). 
diesen relativ eindeutigen Befund könnte darin liegen, daß die zunehmende Verbreitung der Inkubationsheiligtümer, gerade in hellenistischer Zeit, den Akzent auf den Zustand des Schlafens legen ließ, während später in An-

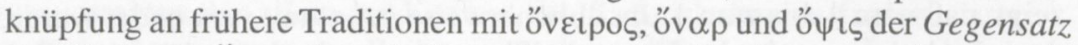
zur Wachwelt, $̋ \pi \alpha \rho$, bzw. die Priorität des Visuellen betont werden sollte. ${ }^{33}$

Bis auf Artemidor von Perge wurden bislang keine Beispiele aus dem Umkreis einer intellektuellen Elite gegeben ${ }^{54}$ Es überrascht freilich nicht, daß sich gerade die hellenistischen Dichter wiederholt des Motivs bedienten: Kallimachos hat zu Beginn seiner Aitia offenkundig die Dichterberufung des Hesiod zu einem Traum umgestaltet; für Herodas spielt ein langer Traumbericht im 8. Mimiambos in der Auseinandersetzung um die neue Dichtungsart die zentrale Rolle; bei Apollonios von Rhodos haben mehrere der wichtigen Protagonisten in den Argonautika Träume; Moschos läßt seine Europa mit einem Traum der gleichnamigen Hauptperson beginnen; ${ }^{55}$ und einige der auf Papyrus neugefundenen Epigramme des Poseidippos haben Träume, ihre Deutung und Erfüllung zum Inhalt. ${ }^{56}$ Schließlich sei an die in den Komödien des Plautus erhaltenen Reflexe griechischer Vorbilder erinnert, in denen gelegentlich auch längere Traumpartien zu finden waren. ${ }^{57}$ Angesichts der Überlieferungslage fällt es schwer, Aussagen über die $\mathrm{Zu}$ oder Abnahme in der literarischen Verwendung von Träumen gegenüber

${ }^{53}$ In diesem Sinne interpretiert auch Lazzarini, Iscrizioni (wie Anm. 16), S. 853, den Befund. Auf ein Inkubationsheiligtum (Asklepieion in Sikyon) verweist Paus. 2,10,2 bezüglich der Statuen von Oneiros und Hypnos.

${ }^{54} \mathrm{Im}$ 'Archiv des Ptolemaios' fand sich zwar eine Reihe qualitativ beachtlicher literarischer Texte (hierzu D. J. Thompson, Ptolemaios and the Lighthouse. Greek Culture in the Memphite Serapeum, PCPhS 33 [1987], S. 105-121), Ptolemaios ist damit aber noch nicht zur kulturtragenden Oberschicht zu rechnen.

${ }^{55} \mathrm{Zu}$ Kall. Ait. I Fr. 2 u. 2a-d: L. Lehnus, Bibliographia Callimachea 1489-1988, Genua 1989, S. 63 f. Zu Hdas. Mim. 8,14-66: V. Gigante Lanzara, Il sogno di Eroda, in: G. Arrighetti/ F. Montanari (Hg.), La componente autobiographica nella poesia greca e latina fra realtà e artifico letterario, Pisa 1993, S. 229-239. Zu Apollonios (bes. Arg. 3,616-632; 4,662-671; 4,17311745 und weiteren Stellen): Kessels, Dreams (wie Anm. 4), S. 156 ff. Zu Moschos Eur. 1-27: M. Campbell, Moschus. Europa (Altertumswissenschaftliche Texte und Studien 19), Hildesheim u.a. 1991, S. $21 \mathrm{ff}$.

${ }^{56}$ Die Epigramme in ihrer Gesamtheit sind bislang nur durch eine Vorab-Publikation bekannt: G. Bastiani/C. Gallazzi, Posidippo. Epigrammi, Mailand: Edizioni il Polifilo 1993; drei Epigramme sind offiziell publiziert: G. Bastiani/C. Gallazzi, Il poeta ritrovato. Scoperti gli epigrammi di Posidippo in un pettorale di mummia, Ca' de Sass. 121, 1993, S. 34-39; dazu jetzt M. W. Dickie, An Ethnic Slur in a New Epigram of Poseidippus, PLLS (= Papers of the Leeds International Latin Seminar) 19 (1996), S. 327-336.

${ }^{57}$ Zur römischen Komödie (bes. Plaut. Mil. 380-401, Merc. 225-271, Rud. 593-612, Curc. 246-263): F. Leo, Plautinische Forschungen, Berlin ${ }^{2} 1912$, S. 162 ff.; J. Bayet, La croyance romaine aux présages déterminants: aspects littéraires et chronologie, in: Hommage à J. Bidez et F. Cumont, Brüssel 1949, S. 13-30, bes. 14-21; F. Arnaldi, Sogni ellenistici, SIFC (= Studi Italiani di Filologia Classica) 27/28 (1956), S. 8-11; J. Collart, La 'scène du songe' dans les comédies de Plaute (Remarques sur quelques procédés formels), in: M. Renard/R. Schilling (Hg.), Hommages à Jean Bayet (Coll. Latomus 70), Brüssel 1964, S. 154-160; A. Katsouris, Notes on Dreams and Dream-Like Visions, Dodone 7 (1978), S. 43-86. Terenz gestaltet dagegen keine Traumszenen und verweist nur auf die Möglichkeit des Träumens (für Hinweise danke ich E. Stärk, Leipzig). 
früheren Jahrhunderten zu treffen, doch gewinnt man nicht den Eindruck eines Bedeutungsverlustes, zumal wenn in der Dichtung programmatische Aussagen in Traumform gekleidet werden. ${ }^{58}$

Das aufgezeigte Spektrum wäre jedoch nicht vollständig, wenn abschlieBend nicht nochmals Apollonios, der jüngste Bruder des Ptolemaios, zu Wort käme. In einem Brief an Ptolemaios beklagt er sich geradezu feindselig darüber, den Träumen und der Deutung, wohl des Bruders, gefolgt - und getäuscht worden zu sein:

„Denn du lügst alles [zusammen] und die Götter bei dir gleichfalls, denn sie haben uns in einen großen Schlamm geworfen und worin wir sterben können, und wenn du [im Traume] gesehen hast; daß wir gerettet werden sollen, [gerade] dann werden wir untergetaucht, [...] in die Irre geführt von den Göttern im Glauben an die Träume.“"

Und auf dem verso des Briefes ist neben dem Namen des Adressaten noch ironisch vermerkt: „An die, welche die Wahrheit sagen.“ Seine in einem früheren Brief geäußerte Überzeugung - ,ohne die Götter geschieht nichts“ - hat einen negativen Beigeschmack erhalten. ${ }^{59}$ Die Passage ist wichtig: Sie zeigt, daß erfolgreiche Kommunikation mit den Göttern immer wieder der Bestätigung bedurfte, und: Nicht nur die Epikureer konnten bis zur strikten Leugnung jeglicher Signifikanz und Relevanz von Träumen gehen. Das Interesse an Träumen konnte sich durchaus auch in Kritik und Ablehnung äußern.

\section{IV.}

Dieses Panorama hellenistischer Träumer, Deuter und Traumtexte entbehrt insofern nicht einer quellenbedingten Einseitigkeit, als das meiste Material aus Ägypten stammt; daraus sollte aber nicht der Schluß gezogen werden, $\mathrm{da} ß$ andernorts weniger und weniger überzeugt geträumt und gedeutet wurde, weil man dort weniger auf Träume gab. Dennoch gewähren die Texte einen Einblick in die mentale Welt der hellenistischen Zeit, sowohl in städtischem als auch in privatem Kontext: Träume stießen auf Interesse bei $\mathrm{al}$ len gesellschaftlichen Gruppen, nicht zuletzt bei den Dichtern; ${ }^{60}$ sie veran-

\footnotetext{
${ }^{58}$ Vgl. E. Bächli, Die künstlerische Funktion von Orakelsprüchen, Weissagungen, Träumen usw. in der griechischen Tragödie, Winterthur 1954; Brillante, Studi (wie Anm. 12) passim; S. M. Oberhelman, The Oneirocriticon of Achmet. A Medieval Greek and Arabic Treatise on the Interpretation of Dreams, Lubbock/Texas 1991, S. 25-29.

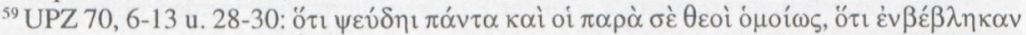

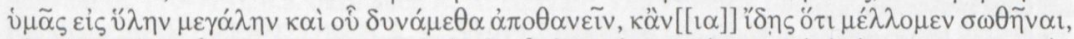

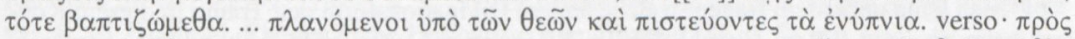

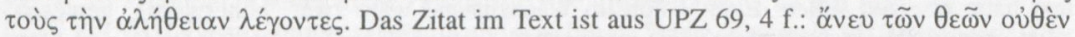

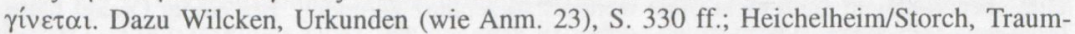
glauben (wie Anm. 23), S. 569, jedoch mit kaum mehr akzeptabler Wertung; Bell, Popular Religion (wie Anm. 23), S. 96; Thompson, Memphis (wie Anm. 27), S. 251 f.; Bulloch, Images (wie Anm. 17), S. 245.

${ }^{60}$ Aufgrund der Quellenlage lassen sich keine Aussagen darüber treffen, ob eine bestimmte Gruppe innerhalb einer Polis oder ein bestimmtes ethisch oder religiös geprägtes Milieu für Träume besonders mental prädisponiert war oder bevorzugt mit ihnen umging.
} 
laßten Menschen aller Schichten zu Handlungen von durchaus beträchtlicher Konsequenz, denkt man etwa an aufwendige Stiftungen oder an den nicht immer billigen Gang zum Traumdeuter, dessen Methoden freilich im Dunkeln blieben. Die Bandbreite der Traummotive und -inhalte reichte dabei von direkten Eingriffen des Göttlichen bis zu einer intensiven Verarbeitung des Alltagsgeschehens im Traum, von deren Rückkopplung in die Wachwelt die Quellen schweigen ${ }^{61}{ }^{1}$ Es liegen somit zwei unterschiedliche Arten von Träumen vor: Zum einen zumindest als klar bezeichnete Aufträge eines Gottes, die erfolgreich als Argument eingesetzt werden konnten, wie das Beispiel des Artemidor von Perge zeigte und das des Zoilos von Aspendos möglich erscheinen läßt. Mit diesen Träumen und mit der Berufung auf sie war die Möglichkeit gegeben, sich anderen gegenüber als besonderer Günstling der Götter darzustellen - nicht jeder dedizierte $\kappa \alpha \tau^{\prime}$ öv $\alpha \rho$ - und eigene Handlungen darauf abzustellen. Man bewegte sich im Rahmen des Alltäglichen, erschloß sich und erklärte diesen Raum - und überschritt ihn gleichzeitig. ${ }^{62}$ Die andere Art von Träumen war symbolischer Art bzw. enthielt Bildfolgen, die eine starke Korrespondenz zur Wachwelt aufweisen. Daß die Träume aus dem 'Archiv des Ptolemaios' geradezu in Form eines 'Tagebuches' aufgeschrieben wurden, hängt vielleicht mit einer Art Selbstvergewisserung zusammen, vielleicht dient die Verschriftlichung auch einfach nur der Kommemorierung für eine spätere Deutung. Den Träumenden war gemeinsam, daß man über seine Träume - zumindest solche, die man für wichtig hielt oder die verunsicherten - sprach oder sie schriftlich niederlegte $;{ }^{63}$ oder man deutete je nach Art des Traumes und eigener Disposition selbst - gelegentlich auch falsch, denkt man an die Reaktion von Ptolemaios' Bruder Apollonios aus dem memphitischen Serapeion. Die Beispiele lassen insgesamt den Schluß zu, daß die Einstellung gegenüber den Träumen und ihrer 'Realität' eine ganz entscheidende Voraussetzung dafür gewesen sein muß, daß Träume, die zu bestimmten Zwecken, etwa der Legitimation, verbreitet wurden, überhaupt auf einen 'bereiteten Boden' fallen konnten. Sie sagen freilich nichts über einen Wandel in der Gottesvorstellung aus bzw. lassen sich nicht im Hinblick auf eine neue Religiosität interpretieren.

$\mathrm{Da}$ auch im erweiterten Poliszusammenhang Träume eine Bedeutung haben konnten, sei abschließend an einer Passage aus der lindischen Anagraphe, die im Jahre 99 v.Chr. in Stein gehauen wurde, vorgeführt: ${ }^{64} \mathrm{Als}$

${ }^{61}$ Auch diese Träume konnten als göttliche Fürsorge interpretiert werden, ohne daß immer ein Gott persönlich agierte.

${ }^{62}$ Man kann außerdem fragen, welche andere Divinationsform den Traum in diesem Kontext hätte ersetzen sollen: Eine Vision im Wachzustand wäre zwar 'höherwertig'. gewesen, hätte aber vielleicht auch nicht ohne weiteres Akzeptanz gefunden. Bei einem Orakel hätte man immerhin noch nachfragen können. Der Vorteil eines Traumes bestand nicht zuletzt darin, $\mathrm{da}$ er nicht nachprüfbar war.

${ }^{63}$ Das Erzählen von Träumen gehörte offenkundig zu den Standardthemen der Geschwätzigkeit ( $\dot{\alpha} \delta \circ \lambda \varepsilon \sigma \chi^{\prime} \alpha$ ), dazu Theophr. Char. 3,2: „Dann erzählt er [sc. der Geschwätzige], welches Traumbild er letzte Nacht gesehen hat."

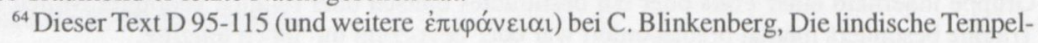


in den Jahren 305/304 Demetrios I. Poliorketes, der Städtebelagerer, vor der Stadt erschien, ,trat die Göttin [sc. Athena] $\kappa \alpha \theta$ ' v̈ $\pi v o v$ an ihn [sc. den Priester Kallikles nach Beendigung seines Amtsjahres] heran und befahl ihm, Anaxipolis, einem von den Prytanen, zu melden, er möge dem König Ptolemaios schreiben" und diesen um Hilfe bitten. ${ }^{65}$ Das nun folgende Erzählsegment ist uns bereits geläufig - woran sich die literarische Konvention gut erkennen läßt: Kallikles ignoriert den Traum und wird erst nach sechsmaliger Intervention der Göttin tätig - Athena hatte offenbar viel Geduld und Interesse am Wohlergehen der bedrängten Rhodier. Ptolemaios sandte Diodor zufolge tatsächlich mehrfach Hilfe und machte sich somit um die Verteidigung der Stadt verdient. ${ }^{66}$ Für die stolze rhodische Lokalüberlieferung stand jedenfalls die durch den Traum angezeigte Wirkmächtigkeit der Göttin im Zentrum, wodurch, ,ein Kompromiß zwischen der hergebrachten Frömmigkeit und den realen Verhältnissen der neuen Zeit" hergestellt war. ${ }^{67}$

chronik (Kl. Texte 131), Bonn 1915 (und Nachdrucke) = FGrHist 532, dazu noch ders., Lindos, Fouilles de l'Acropole 1902-1914. II. Inscriptions, Berlin, Kopenhagen 1941, Nr. 2, S. 185 ff.; A. Chaniotis, Historie und Historiker in den griechischen Inschriften. Epigraphische Beiträge zur griechischen Historiographie (HABES 4), Wiesbaden 1988, S. 52-57 (T 13), bes. Anm. 109; Leuci, Terms (wie Anm. 4), S. 27 f. Zum historischen Kontext vgl. E. Will, Histoire politique du monde hellénistique, Bd. 1, Nancy ${ }^{2} 1979$, S. 70,73 f., vornehmlich basierend auf Diod. 20,81-88 u. 91-100,4. Eine englische Übersetzung bei Lewis, The Interpretation (wie Anm. 23), S. $44 \mathrm{f}$.

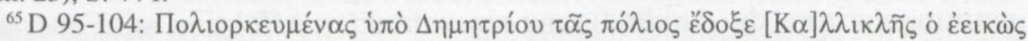

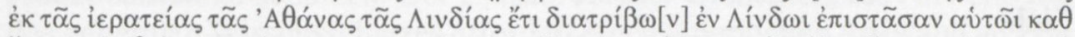

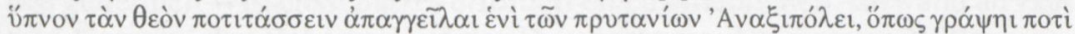

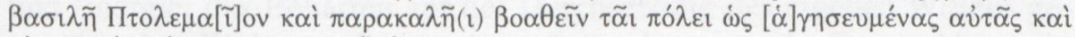

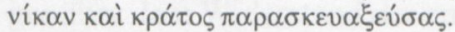

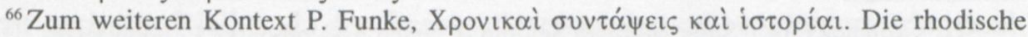
Historiographie in hellenistischer Zeit, Klio 76 (1994), S. 255-262, bes. 257 f. (dort weitere Lit.), auch G. A. Lehmann, Das neue Kölner Historiker-Fragment (P. Köln Nr. 247) und die

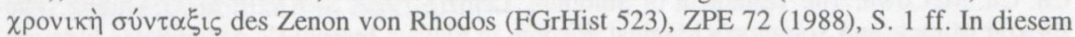
Zusammenhang ist noch eine Weihung eines Ptolemaios, wohl des ersten, in der Tempelchronik relevant (C 110-113), u.a. dazu auch R. A. Hazzard, Did Ptolemy I get his Surname from the Rhodians in 304?, ZPE 92 (1992), S. 52-56; zur Bedeutung von Rhodos für den ersten Ptolemäer vgl. R. M. Berthold, Rhodes in the Hellenistic Age, Ithaca 1984, S. 78 mit Anm. 40.

${ }^{67} \mathrm{Vgl}$. C. Habicht, Gottmenschentum und griechische Städte (Zetemata 14), München ${ }^{2} 1970$, S. 109 f., 233 f. (dort das Zitat), der von einer Erfindung der Priester ausgeht, die ,aber wohl von keinem Rhodier wirklich geglaubt" wurde. Zum Prestige für die Städte vgl. R. Lane Fox, Pagans and Christians, London u.a. 1986, S. 122. Daß die lindischen Texte nicht singulär sind,

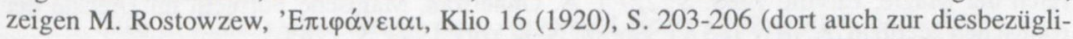
chen Geschichtsschreibung), W. K. Pritchett, The Greek State at War, Part III: Religion, Berkeley u.a. 1979, S. 11-46, u. Leuci, Terms (wie Anm. 4), S. 218 ff. 